International Journal of Computer Engineering \& Technology (IJCET)

Volume 10, Issue 2, March-April 2019, pp. 258-269, Article ID: IJCET_10_02_025

Available online at

http://iaeme.com/Home/issue/IJCET?Volume=10\&Issue $=2$

Journal Impact Factor (2016): 9.3590(Calculated by GISI) www.jifactor.com

ISSN Print: 0976-6367 and ISSN Online: 0976-6375

(C) IAEME Publication

\title{
IMPLEMENTATION OF STABLE PRIVATE CLOUD USING OPENSTACK WITH VIRTUAL MACHINE RESULTS
}

\author{
Nikhil Wagh, Vikul Pawar and Kailash Kharat \\ Department of Computer Science and Engineering \\ Government College of Engineering, Aurangabad, Maharashtra, India
}

\begin{abstract}
In today's era educational organization strongly needs devices which are ready to access and use and also various operating system platforms are required for different learning courses. To achieve this type of environment hardware availability come in front as an important issue along with lots of money required to purchase them. Many educational courses required to run particular software or application on particular operating system platform along with specific hardware configuration. The maintenance of this different versions of operating systems and their installation stuff is hectic process and also required man power. To overcome all this problem there is solution called Cloud OpenStack. Cloud OpenStack allows us to develop an environment on commodity hardware or on existing system present in the educational organization. It's easily handle different version of OS platforms and also monitor and maintain them. Another important thing is many educational organizations still used Virtual machine method to used different OS platforms for various learning courses. This paper come up with implementation of Cloud OpenStack in Educational Organization and analyzing the results. The outcomes of Cloud OpenStack compare with the Virtual Machines method and find out which technique is better and more suitable for academics.
\end{abstract}

Keywords: Private Cloud, Educational Organizations (EOs), OpenStack, Virtual Machine Instance (VMI), Cloud Computing (CC), OpenStack Educational Cloud (OEC).

Cite this Article: Nikhil Wagh, Vikul Pawar and Kailash Kharat, Implementation of Stable Private Cloud using OpenStack with Virtual Machine Results, International Journal of Computer Engineering and Technology, 10(2), 2019, pp. 258-269 .

http://iaeme.com/Home/issue/IJCET?Volume=10\&Issue=2

\section{INTRODUCTION}

In this modern era the technologies in the IT field are changing and improving so rapidly. So it's difficult for many educational organizations (EOs) to purchase hardware resources which meets with new technology [1]. Hence they finding the different ways to overcome this 
problem and they found the solution called Cloud Computing but they don't know how to implement this technology as per organizational networking environment. Cloud Computing is known as sharing of information, software and applications over the internet [2]. CC easily fulfill all the problems such as providing the resources as per the requirements, less money required because it's built on commodity hardware, easy to use and maintain, flexible and scalable, easy to upgrade as per the latest technological needs [1]. CC is an umbrella of technologies which include Grid Computing, Virtualization, Utility Computing, Internet and Web Services [3]. Virtualization is the heart of CC which helps to run multiple OSs at the same time on single machine but many EOs still working on traditional method called Virtual Machine method because they don't know how to setup cloud in their network and how we can use them. Virtual Machine have some boundaries on hosting limited number of OSs and it's also take time to deploy ISO images because you have to download, install and configure the OS manually. Apart from this Virtual Machine required high configured system to run Virtual instances smoothly otherwise it takes time to load and run the processes [4]. Hence it's important for EOs to adopt new technology such as Cloud Computing (CC) to overcome many problems.

Now we can moving towards the deployment of $\mathrm{CC}$ as per our educational infrastructure is working and there are many open source cloud frameworks are present to deploy the cloud such as OpenStack, Cloud Stack, Nimbus, Open Nebula, Cloud Foundry and many more [2]. But we are implementing our cloud infrastructure by using OpenStack framework. It is better framework then other once. It's an open source and supports both private and public cloud environment. OpenStack is easy to deploy, more scalable and compatible for EOs [1] [2]. In 2010, Rackspace and NASA togetherly work on project called OpenStack. It provides many services and at basic level it configure on 2 nodes Controller and Compute nodes. We can extend services and power of cloud as per organizational requirements [5] [2].

Let's study some cloud services which are already using some organization form third parties.

\section{LITERATURE SURVEY}

In the beginning of cloud computing phase many US based EOs believe on cloud capabilities and they started using some cloud services for their students like email facilities and storage facilities and later on cloud computing started capturing the IT market. Now in India, many EOs uses cloud services provided by third parties like Amazon AWS, IBM, and Google Cloud Services etc. This cloud providers charge the money as per the use of cloud services. You can even pay money based on your use of resources. Again some EOs uses CC for hosting academics learning management system which includes use of Moodle for conducting exams then universal compiler for program compilation and many more[8]. The performance of this is depend upon the configuration of cloud instances provided by the third parties [6]. The architecture of services provided by third parties as shown below. 




Figure 1 Third Parties Cloud Provider Architecture

In Figure 1, we can see that EOs request for services to the Third Parties then Third Parties request for servers to fulfill the EOs needs and if the servers are available to provide then it instantly provided to the Organization.

The most important things while getting services from third parties, that is organizations have to pay money for services and also they rely on cloud provides for everything like taking more services or reducing it or any problem occur in networking every time you have to contact to the cloud customer support. Due such problems taking services from third parties it's not affordable for EOs. Hence OpenStack cloud allows organizations to build their own cloud framework in educational environment and increase and decrease the power or capacity of the cloud whenever they required by adding and removing machines form the cloud network. OpenStack cloud framework is best suited framework for organization [2].

\section{PROPOSED SYSTEM ARCHITECTURE}

\subsection{OpenStack Architecture}

Our proposed system architecture is based on OpenStack framework. This framework is depend on the networking environment of EOs. First we will see the OpenStack architecture which includes various modules such as Horizon, Swift, Nova, Glance and Keystone and many more [4] [5].

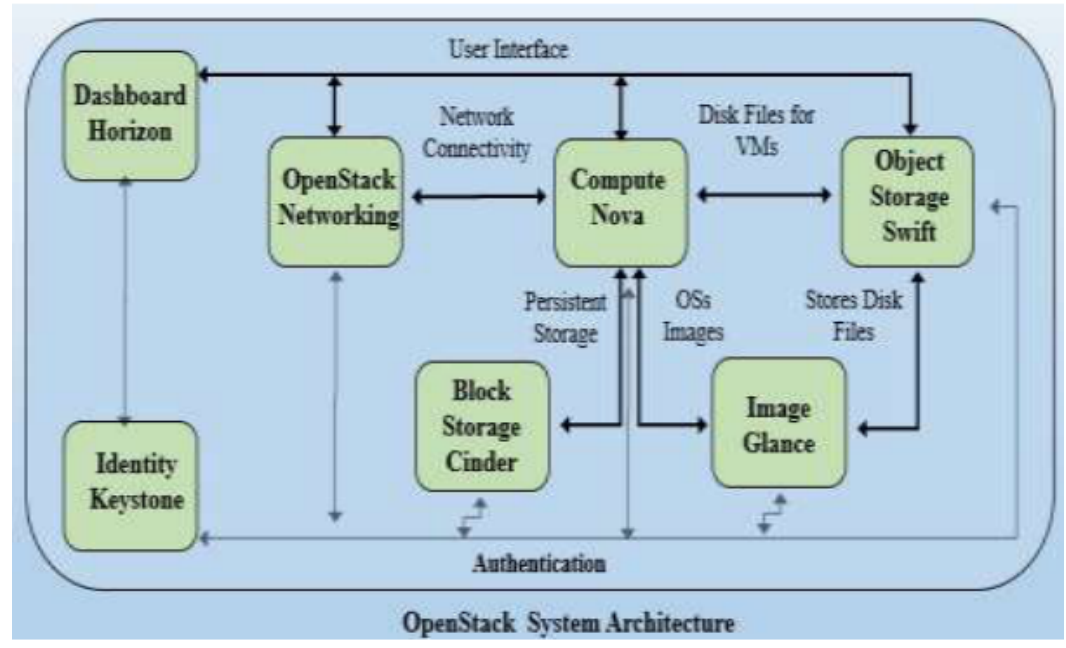

Figure 2. OpenStack System Architecture 
1. Dashboard (Horizon): It provides GUI to the users to handle the cloud and perform some task like manage and monitor the instances, create key pair for security, attached or detached storage or volume to the instances and many more [9].

2. Networking (Neutron): User can create our own network on cloud and connect nodes or servers to the one or more network present in the organizations [4].

3. Block Storage (Cinder): User can attached or detached Virtual Hard Drive to the instances. Also take backups of every instance via creating replications [4].

4. Compute (Nova): It handles the Virtual Machine created on cloud. It handles creations, start and stop operations of instances and many more [9] [4].

5. Image (Glance): It's like an index of Virtual Disk Images with which we can do creating snapshots as a backup and use whenever you want. Supported image formats are QCOW2, VHD, ISO, VMDK, VDI [4].

6. Object Storage (Swift): Provides web accessible storage system for handling huge data which includes Images, Videos, Email Messages, Virtual Images and Files [4].

\subsection{Cloud Architecture for EOs}

We saw the OpenStack framework for our organization now we are going to see whole cloud architecture as per our educational environment.

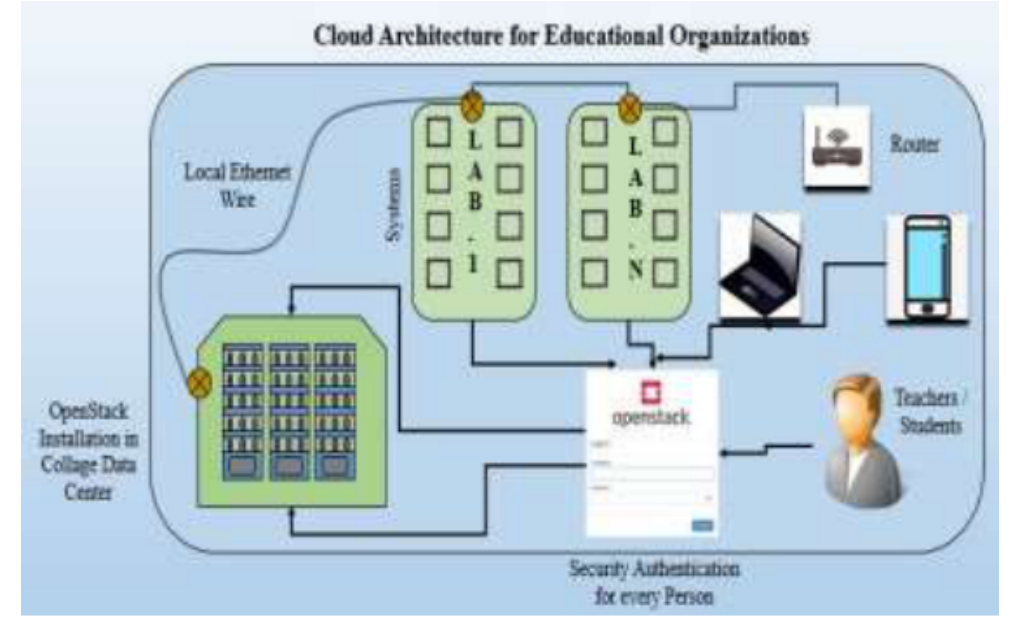

Figure 3. Cloud Architecture for EOs

Figure 3 shows that private cloud for EOs. This cloud is going to develop on blade servers present in the college data center or we can also implement on commodity hardware without spending any money on buying high configured machines. Then students or teachers use this cloud in the Labs through the local network in their campus or they can also use on their laptops or on mobiles through the router configured in the same network. This cloud setup is limited to the single organization and only the students and teachers are present in that organization are authorized to access cloud services. Every student or teacher must go through authentication process for security purpose. We can make this cloud public also to use for other organizations but due to privacy concern we prefer the Private cloud.

\section{IMPLEMENTATION}

We saw the architecture of OpenStack and Cloud environment in proposed system section. Now we are going to implement OpenStack framework on Ubuntu 18.04 Operating System. We implement this framework on servers called Controller node and Compute node. The minimal requirements of this implementations required at least two servers or nodes. Figure 4 
shows the implementations architecture of OpenStack Cloud. Controller node handles the Keystone, Horizon, Glance and many more and Compute node handles Nova services. This two servers communicates with each other via neutron service over Internal network and External network is used for applications which are ready to access on cloud [3] [9].



Figure 4. OpenStack Implementation Architecture

\subsection{Controller Node}

The installation of controller node starting with update and upgrade command. But before starting implementation make sure that the controller node must have two NIC cards. First NIC address save as a static IP and other one save as DHCP and then start working with first database service is SQL service and then step by step Message queue and RabbitMQ services. Then NTP service synchronizes the services among servers. Other servers follow the controller node for synchronization.

All the information entered while installation of OpenStack is stored in SQL DB service, Message queue service work as message broker between machines. Once we implement the basic services then remaining services Keystone, Glance, Nova, Neutron and Horizon are installed step by step. This services are mandatory for installation of cloud. Apart from this we can install other services as per our need like Orchestration, Block Storage, Telemetry and many more [3] [9]. Figure 5 shows the services comes under controller node [10].

\subsection{Compute Node}

Compute node works on Nova service which is important and used to handle the instances on cloud framework. RAM, Storage and Maintenance of instances is handle by compute node. By default Hypervisor of compute node is KVM. It also work on networking for communication to the controller node. The power of cloud in terms of processing and storage increasing by increasing more compute nodes in the same network. The Figure 5. Shows the services comes under Compute node [3] [9]. 


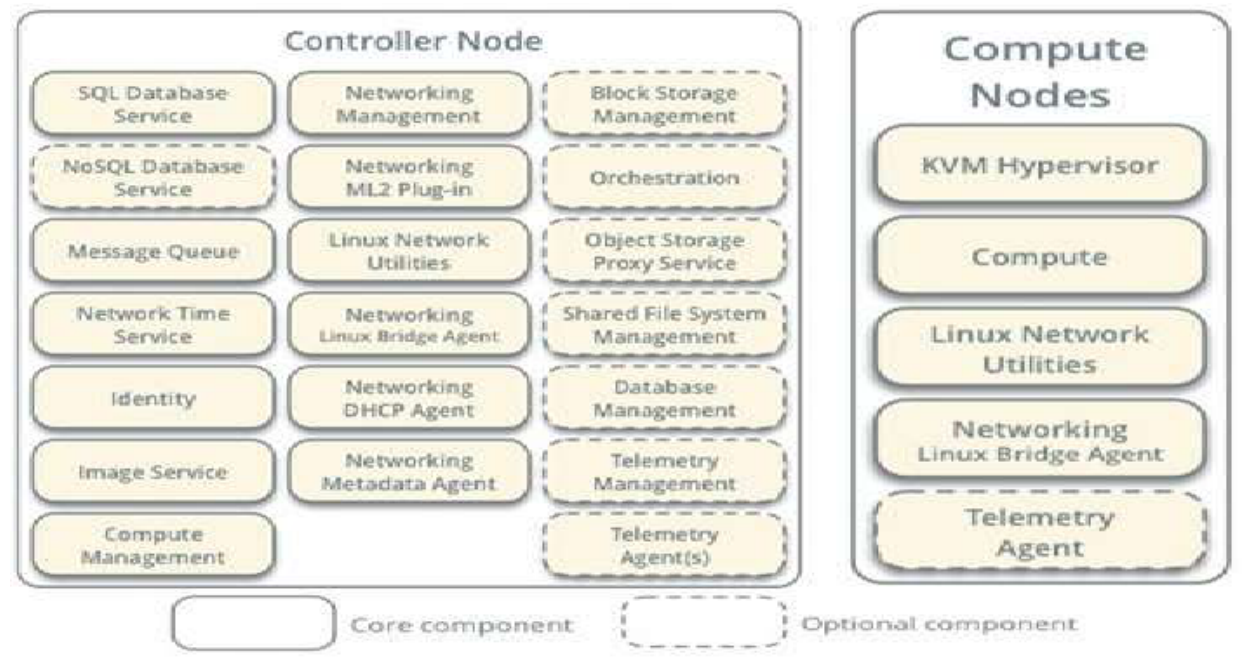

Figure 5. Services comes under Controller and Compute Node

Once we done with successful installation of controller and compute node we got the dashboard of OpenStack Cloud from where we can manage our cloud framework. Then we launch an instances on cloud and provide to the students for their learning courses. Figure 6. Shows the dashboard of OpenStack Cloud.

When the cloud is setup in organizational environment then we can easily provide IAAS, PAAS and SAAS services to the user. Due to this easy way of installation and proper utilization of hardware and better services provided by cloud OpenStack is the great learning methodology for students.

\section{EXPERIMENTAL RESULTS AND PERFORMANCE ANALYSIS}

After the stable cloud setup user can launch instances, deal with various images and projects then also use various flavors of images to launch the services via OpenStack dashboard [9].

1. OpenStack Login Dashboard

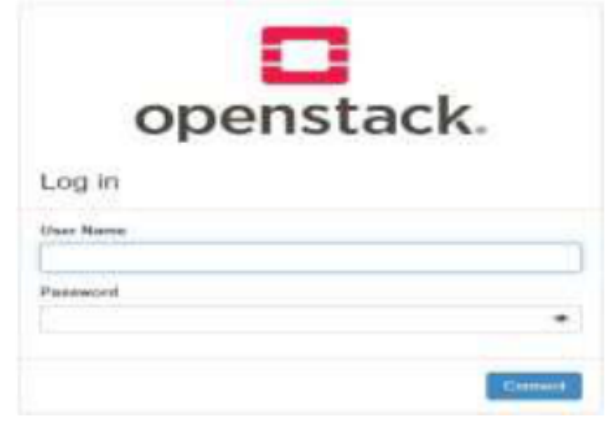

Figure 6. Cloud OpenStack Dashboard

All important activities for handling cloud is done from this dashboard. Figure shows the Login page of dashboard. We can access this dashboard using the IP address of controller node For example.

http://<Controller IP_address $>/$ dashboard.

\section{Flavors and Images}

OpenStack by default provide a preconfigured list of flavors and also with this we can create our own flavors as per our need. Use that flavors at the time of creation of instance. This 
flavors contain the combination of RAM, Storage, VCPU and many more data which is useful to run instances.

\section{Key Pair}

Due to the use of Key Pair only authorized user can get the access of instance on cloud.

\section{Overview}

It shows the how much RAM, Storage, Floating IP's and system related data are currently used in cloud. According to them you can increase and decrease the power of cloud.

\subsection{Performance Analysis}

After successful installation of OpenStack Educational Cloud (OEC) now we are ready to launch instances on cloud platform and perform the operations to check which instances perform better in a minimum RAM and Storage and gives the better results. We are comparing our OEC instance results with Virtual Machine instance (VMI). In educational system to launch VMI, students or teachers uses some common platform called VMware, Oracle VirtualBox and many more. To compare OEC instance results with VMI results, we launch one instance on Cloud platform and another one on Oracle VirtualBox. The configuration of both the instances are 2GB RAM, 30GB Hard Drive and 1 VCPUs with common data sets which we are going to apply on instances to check the performance and execution time.

We launched Ubuntu 18.04 operating system on both the platforms and for simplicity we taken the word and character count program in $\mathrm{C}$ language and execute that program through GNU Compiler Collection (GCC compiler). We applied this program on the different sizes of Text Files like 50KB, 500KB, 1MB, 5MB and many more and we got the results which are shown in following Figure 7.

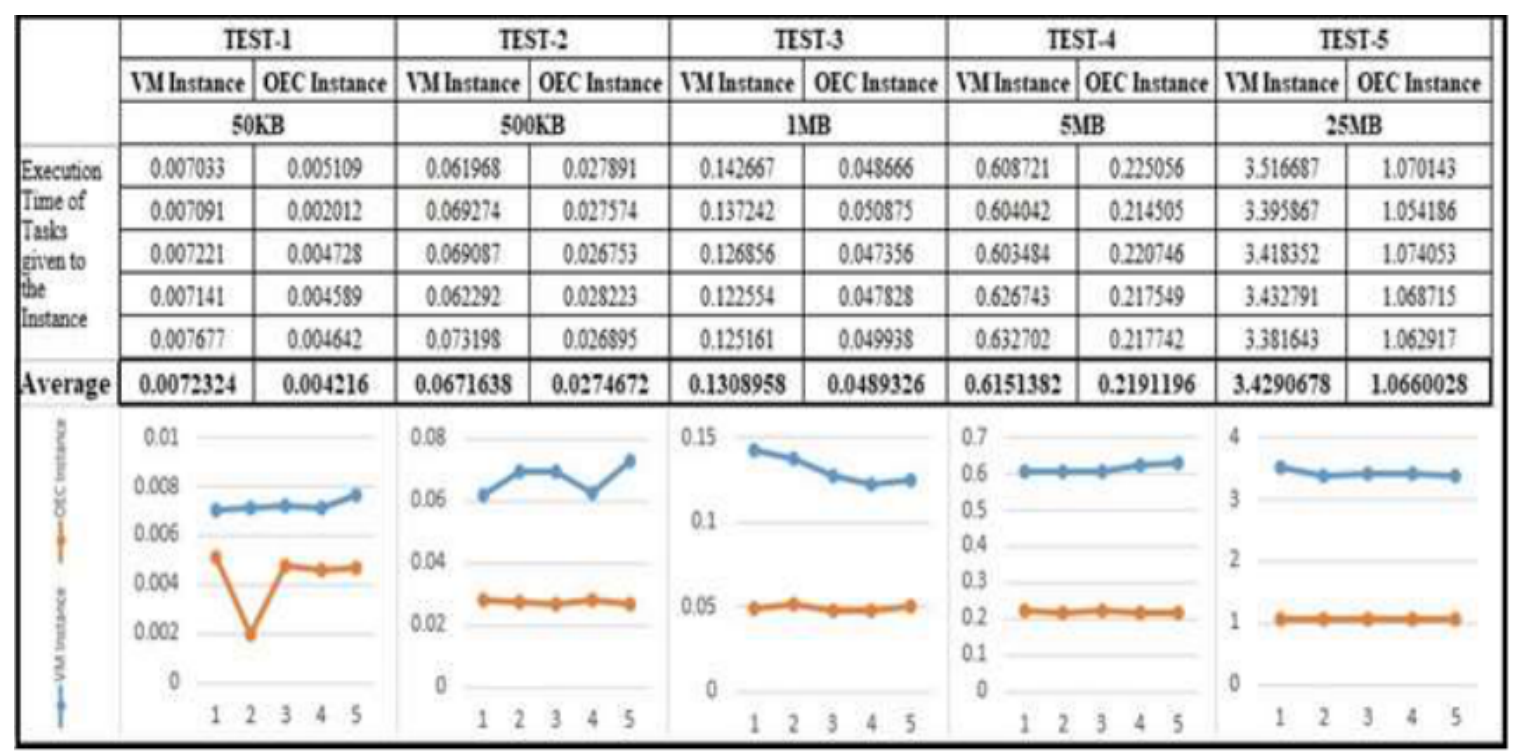

Figure 7. Execution Time of the Tasks in both the Instances.

Figure 7 shows that the time taken by VMI and OEC instance to execute the Text files with different File Size. The time required for both the instances to execute same file clearly shown in the charts. Blue lines indicates the VMI results and Orange Lines indicates the OEC instance results. X-Axis shows the number tasks and Y-Axis shows the Time Lag. We can easily identity that OEC instance take very less time to execute same operation performed by VMI. 


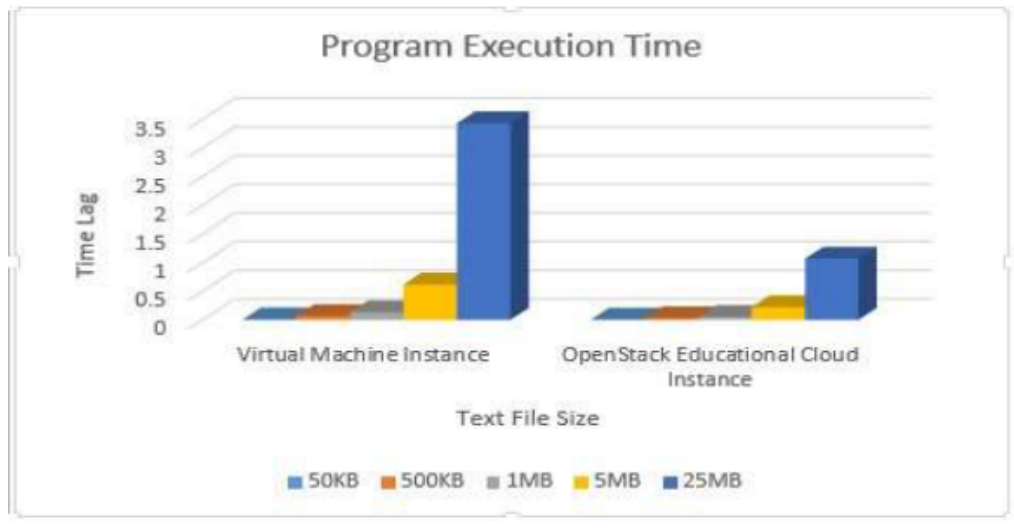

Figure 8. Program Execution Time Chart (Based on Average shown in Fig 7)

To get more details about the execution time we calculate the average of each file size separately in both the instances and we got the results shown in Figure 8.

Figure 8 shows that the average time required for both the instances to execute same task. When the File Size is increasing VMI execution time is also increasing and due to this reason it's not at all suitable for large data sets. Let's see the time spent by CPU on every task performed by both the instances.

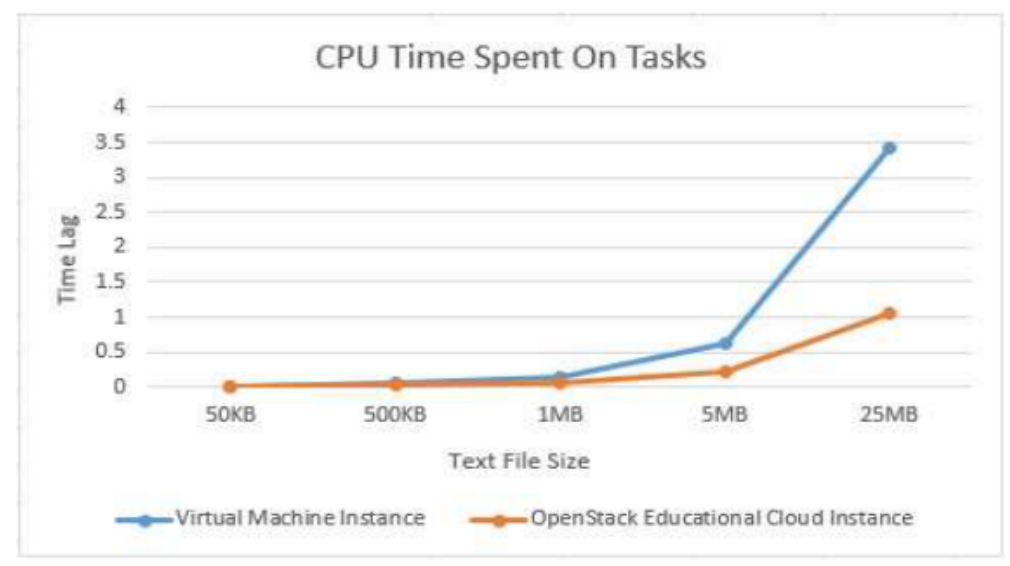

Figure 9. CPU Time Spent on Each Task.

Figure 9 shows that CPU in VMI spent more time to execute the task as compare to OEC instance. Along with the more time, VMI also facing system hanging problem and work slow as compare to OEC instance. OEC instance work far better than VMI because it's properly utilize the hardware resources to perform the task. Now, let's compare the Processing Time taken by both the instances.

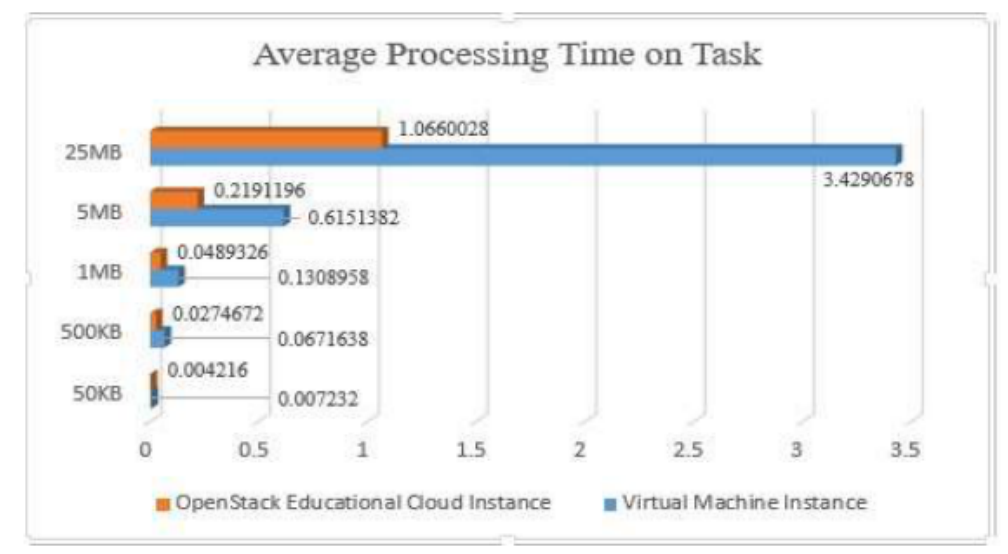

Figure 10. Average Processing Time required for Instances 
Figure 10. Shows that the, average time taken by instances to process the tasks and we can see that the time required for VMI to process the data is higher than the OEC instance. To know about the system performance let's check the health of both the instances.

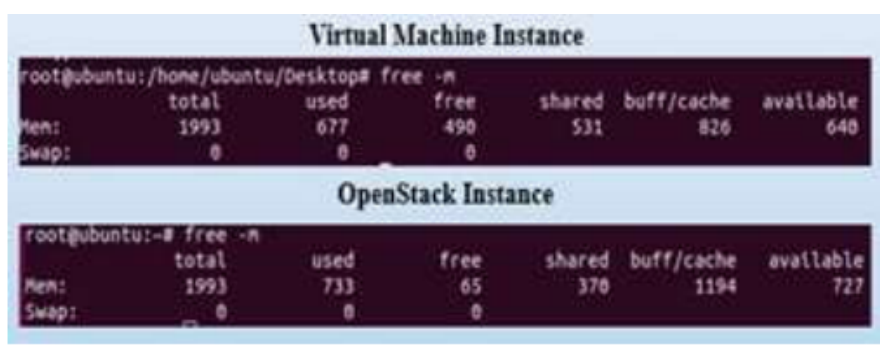

Figure 11 Available Memory on Instances

Figure 11 shows that the how much memory is available after performing operations. We see that the cache memory in OpenStack Instance is higher than the VMI hence it perform recent operations from buffer memory and reduce the execution time.

\begin{tabular}{|c|c|c|c|}
\hline \multicolumn{2}{|c|}{ Virtual Machine Instance } & \multicolumn{2}{|c|}{ OpenStack Instance } \\
\hline rooteubuntu:/hom & g/ubuntu/Desktop" cat /proc/memtnfo & rootgubuntu: $-\# \mathrm{C}$ & at /proc/mentnfo \\
\hline MemTotal: & $2041316 \mathrm{kB}$ & Memiotal: & $2041260 \mathrm{kB}$ \\
\hline Memfree: & $474096 \mathrm{kB}$ & MemFree: & $75812 \mathrm{kB}$ \\
\hline MemAvatlable: & $650792 \mathrm{kB}$ & MemAvatlable: & $745332 \mathrm{kB}$ \\
\hline Buffers: & $62268 \mathrm{kB}$ & Buffers: & $330500 \mathrm{kB}$ \\
\hline Cached: & $773784 \mathrm{kB}$ & Cached: & $816120 \mathrm{kB}$ \\
\hline SwapCached: & $0 \mathrm{kB}$ & SwapCached: & $0 \mathrm{kB}$ \\
\hline Active: & $834276 \mathrm{kB}$ & Active: & $1070720 \mathrm{kB}$ \\
\hline Inact tve: & $595156 \mathrm{kB}$ & Inactive: & $746712 \mathrm{kB}$ \\
\hline Active(anon): & 699008 k8 & Active(anon): & $712136 \mathrm{kB}$ \\
\hline Inactive(anon): & $438968 \mathrm{kB}$ & Inactive(anon): & $337804 \mathrm{kB}$ \\
\hline Active(file): & $135268 \mathrm{kB}$ & Active(file): & $358584 \mathrm{kB}$ \\
\hline Inactive(ftle): & $156188 \mathrm{kB}$ & Inactive(ftle): & $408908 \mathrm{kB}$ \\
\hline Unevtctable: & $16 \mathrm{kB}$ & Unevtctable: & $16 \mathrm{kB}$ \\
\hline Mlocked: & $16 \mathrm{kB}$ & MLocked: & $16 \mathrm{kB}$ \\
\hline SwapTotal: & $\theta \mathrm{kB}$ & Swaptotal: & $0 \mathrm{kB}$ \\
\hline Swapfree: & $0 \mathrm{kB}$ & SwapFree: & $0 \mathrm{kB}$ \\
\hline otrty: & $\theta \mathrm{kB}$ & Dirty: & $0 \mathrm{kB}$ \\
\hline Writeback: & $\theta \mathrm{kB}$ & Writeback: & $0 \mathrm{kB}$ \\
\hline AnonPages: & $593448 \mathrm{kB}$ & AnonPages: & $670840 \mathrm{kB}$ \\
\hline Mapped: & $80164 \mathrm{kB}$ & Mapped: & $136428 \mathrm{kB}$ \\
\hline Shmen: & $544580 \mathrm{kB}$ & Shmem: & $379128 \mathrm{kB}$ \\
\hline Slab: & $66016 \mathrm{kB}$ & Slab: & $93156 \mathrm{kB}$ \\
\hline SReclatnable: & $33732 \mathrm{kB}$ & SReclainable: & $67312 \mathrm{kB}$ \\
\hline
\end{tabular}

Figure 12. Dynamic Information of System present in meminfo file.

Next method to check memory performance is vmstat. This method shows that the how properly Cloud OpenStack utilize the resources and gives the better performance as compare to VMI. Figure 13 shows the memory performance.

The Figure 13 shows that the total memory, used memory, active and inactive and many more memory related data. From which we can identify on what level memory is active and used to perform the task. Active memory is used by processes and Inactive memory is allocated to processes but no longer running. Here we can see that OEC instance used memory is more than the VMI memory because the active memory of OEC instance is greater than the VMI memory and out of that how much system is using to perform task. If we closely analyze the active and used memory in the same instance for example VMI active memory is $833076 \mathrm{~KB}$ and out of that the $697432 \mathrm{~KB}$ is used for operations and it's close to the active memory boundary. Because of this, system works slowly and takes time to execute the program. In other side OEC instance active memory and used memory there is valuable difference is present to work system more efficiently. Another important thing is, inactive memory allocated to processes is higher in OEC instance as compare to VMI which helps to execute the program in less time. 


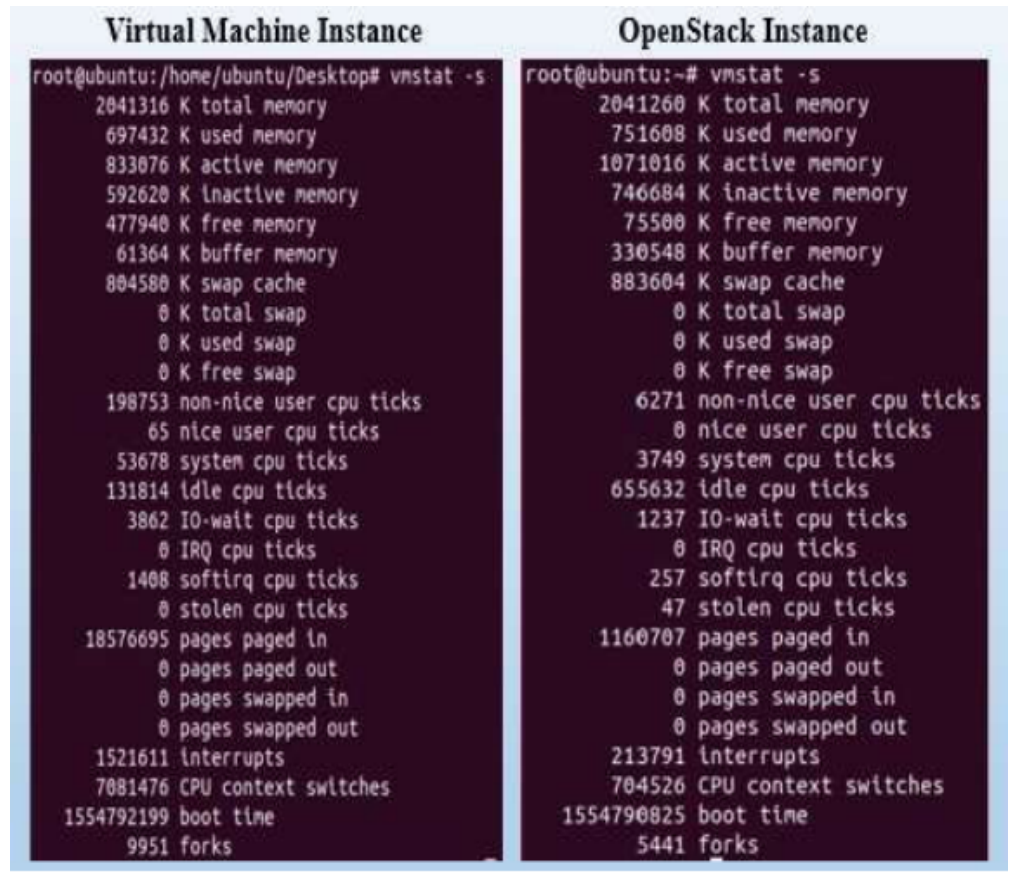

Figure 13. Memory Statistics

After performing thousands of tests on both the instances by using different file sizes we got same result OEC instances take very less time to execute the program. Also perform same operations on high configured instances like 3GB RAM, 50GB Hard Drive, 1 VCPUs and 4GB RAM, 50GB Hard drive and 2VCPUs. Every time we got the OEC instance is far better than VMI hence we can easily use Cloud OpenStack in EOs for proper utilization of resources and getting good results.

\section{CONCLUSIONS}

In this paper we found out best framework for implementing cloud is called cloud OpenStack. OpenStack is powerful enough to fulfill user organizational requirements. Then implement the cloud in organizational network and create Cloud environment. We perform some operation like creation of image, instance and flavors, create key pair for security purpose and many more.

We compare OpenStack cloud instance with traditional Virtual Machine instance and analyze the results and we got the best method for organization which is Cloud Computing method. Student and teachers easily use this instances on cloud and got the better results. It's easy to use, fast, reliable and scalable platform. It contains backup for instance failure, data is stored and maintain properly. Hence it's high time to move EOs towards the Cloud infrastructure in the organization. Now every EOs implement this framework easily and used this cloud services for their student learning courses.

We are succeed with implementation of cloud in our Educational Organization. Now, in future we try to customized and create such a cloud which is useful for other Organizations also like Government Sectors, Small Scale Industries, Private Offices and many more and we also try to provide more security to the access of cloud from anywhere.

\section{ACKNOWLEDGMENTS}

We would like to thank Dr. P. B. Murnal. Dr. V. P. Kshirsagar, Dr. M. B. Nagori for their valuable support and guidance and Department of Computer Science and Engineering, GECA, Aurangabad for providing healthy environment for research work. 


\section{REFERENCES}

[1] Shubham Awasthi, Anay Pathak and Lovekesh Kapoor, "Openstack- Paradigm Shift to Open Source Cloud Computing \& Its Integration", $2^{\text {nd }}$ International Conference on Contemporary Computing and Informatics (ic3i), pp. 112-119, IEEE 2016.

[2] Nikhil Wagh, Vikul Pawar and Kailash Kharat, "Educational Cloud Framework - A Literature Review on Finding Better Private Cloud Framework For Educational Hub", International Conference on Emerging Technologies in Computer Engineering: Micro services in Big Data Analytics(ICETCE), Jaipur, India, Springer 2019.

[3] P.Sai Sheela and Monika Choudhary, "Deploying An OpenStack Cloud Computing Framework for University Campus", International Conference on Computing, Communication and Automation (ICCCA), pp. 819-824, IEEE 2017.

[4] Gaurav Bhatia, Ismail Al Noutaki, Sheikha Al Ruzeiqi and Jawaher A1 Maskari, "Design and Implementation of Private Cloud for Higher Education using OpenStack", Majan International Conference, IEEE 2018.

[5] Jaison Paul Mulerikkal and Yedhu Sastri, "A Comparative Study of OpenStack and CloudStack", $5^{\text {th }}$ International Conference on Advanced in Computing and Communications, IEEE 2015.

[6] E. Krelja Kurelovic, S. Rako and J. Tomljanovic, "Cloud Computing in Education and Student's Needs",MIPRO, pp. 726-731, 2013.

[7] Sultan, N., Cloud Computing for education: A new dawn?, International Journal of Information Management, no.30,2010, link-

https://www.sciencedirect.com/science/article/pii/S0268401209001170

[8] Cloud Computing in Education, IITE Policy Brief, UNESCO, 2010, link https://iite.unesco.org/files/policy_briefs/pdf/en/cloud_computing.pdf

[9] Girish L S and Dr. H S Guruprasad, "Building Private Cloud using OpenStack", International Journal of Emerging Trends \& Technology in Computer Science (IFETTCS), pp. 142-145, 2014.

[10] Services Comes under Controller and Compute Node. Link https://docs.openstack.org/install-guide/overview.html

\section{AUTHOR DETAILS}

Research Scholar Nikhil R. Wagh: I have completed B.E. in 2017 and currently doing M.E. from Government College of Engineering Aurangabad. I have published a paper "Educational Cloud Framework - A Literature Review on Finding Better Private Cloud Framework For Educational Hub", International Conference on Emerging Technologies in Computer Engineering: Micro services in Big Data Analytics (ICETCE), Jaipur, India, Springer 2019.Interested research area is Cloud Computing and I am working on it from last 2 years.

Prof. Vikul J. Pawar: Received the B.E.degree in Computer Science and Engineering from Babasaheb Naik College of Engineering, Pusad under Sant Gadge Baba Amravati University, Amravati, Maharashtra, India, in 2006, and the M.Tech. degree in Computer Science and Engineering from Government College of Engineering, Aurangabad under Dr. Babasaheb Ambedkar Marathwada University, Aurangabad, Maharashtra, India, in 2014. He has 2 years 
of experience in software development in Capgemini Pvt. Ltd. India during 2007-09. He has joined as regular faculty in Government College of Engineering, Aurangabad at the rank of an Assistant Professor in 2011to till date. His research and teaching interests center on the Image Processing, Data Mining \&amp; warehousing and Big Data Analytics. He worked as a reviewer of the International conference ICRTRES at Malkapur in 2015-16. He is a frequent author of IEEE and Springer conference papers.

Prof. Kailash D. Kharat: Received the B.E. degree in Computer Science and Engineering from Government College of Engineering, Amravati under Sant Gadge Baba Amravati University, Amravati, Maharashtra, India, in 2009, and the M.E. degree in Computer Science and Engineering from Government College of Engineering, Aurangabad under Dr. Babasaheb Ambedkar Marathwada University, Aurangabad, Maharashtra, India, in 2012. Now Pursuing PHD from JJTU University Rajasthan In 2013. He joined the faculty of the MSS College of Engineering, Jalna at the rank of an Assistant Professor in 2012-2015. In 2015 he joined the faculty of the Government College of Engineering, Aurangabad, and Maharashtra, India at the rank of an Assistant Professor. His research and teaching interests center on the Data Mining \&amp; warehousing and Big Data Analytics. He worked as an reviewer of the International conference ICRTRES at Malkapur in 2013, 2014, 2015 and 2016. He also received the GATE Scholarship. He is a frequent author of IEEE journal and conference papers. 